\title{
Memory for thematically arousing events
}

\author{
CARA LANEY \\ University of California, Irvine, California \\ and \\ HANNAH V. CAMPBELL, FRIDERIKE HEUER, and DANIEL REISBERG \\ Reed College, Portland, Oregon
}

\begin{abstract}
Many studies have indicated that emotional arousal improves memory for the center, or gist, of an event but undermines memory for the event's periphery. However, all of these studies have elicited emotion by showing participants some salient visual stimulus intended to arouse them (e.g., the sight of a wound). This stimulus may have served as an attention magnet, and this, not the arousal, may have been the cause of the observed narrowing of memory. In this article, we examine how participants remember events that involve thematically induced arousal, arousal produced by empathy, rather than by a visual emotional stimulus. The data show that emotionality improves memory for all aspects of these events, with no memory narrowing.
\end{abstract}

How do we remember the emotional events we experience? Many studies have indicated that emotionality has two effects on memory: First, emotion seems to improve memory for the gist of an event and, also, for details closely associated with this gist (e.g., the exact appearance of characters central to the plot or the details of objects that were perceptually prominent within the scene). Second, emotion seems to undermine memory for more peripheral aspects of the event, including any details not closely tied to the event's main actors or objects and, also, details spatially in the background (see, e.g., Burke, Heuer, \& Reisberg, 1992; Christianson \& Loftus, 1987, 1990, 1991; Wessel \& Merckelbach, 1997; see Reisberg $\&$ Heuer, 2004, for a review).

This dual effect is typically attributed to the effects of arousal per se, with the logic of the claim often referred to as the Easterbrook hypothesis (after Easterbrook, 1959). The idea, broadly, is that arousal (in virtually any form) causes a decrease in the range of cues an organism can take in. This narrowing of attention leads directly to the exclusion of peripheral cues, and this is why emotionality undermines memory for information at the event's edge. At the same time, this narrowing allows a concentration of mental resources on more central materials, and this leads to the beneficial effects of emotion on memory for the event's center. ${ }^{1}$

As we have noted, many studies seem to confirm these claims, but one might raise questions about the general-

Experiment 1 was completed as part of the second author's undergraduate senior thesis; Experiment 2 was completed as part of the first author's senior thesis. We thank Robert Greene, Kerri Pickel, and two anonymous reviewers for their helpful comments on an earlier draft of this article. Correspondence concerning this article should be addressed to D. Reisberg, Psychology Department, Reed College, Portland, OR 97202 (e-mail: reisberg@reed.edu). izability of the available evidence. This concern arises largely because of how the relevant studies have produced emotional arousal. Unquestionably, researchers in this domain have been constrained by the fact that it is often difficult to arouse the university undergraduates who serve as research participants. Researchers have therefore turned to a technique that does reliably produce arousal-namely, the presentation of shocking visual stimuli. For example, several studies have probed participants' memory for a story in which a young boy is involved in an automobile accident; the stimulus sequence includes a photograph of the boy's severed (and then reattached) legs (e.g., Adolphs, Tranel, \& Denburg, 2000; Burke et al., 1992; Cahill \& McGaugh, 1995). Other studies have been performed to examine memory for a sequence that shows a young man who has been shot and is bleeding from his eyeball (e.g., Loftus \& Burns, 1982). In another study, participants were presented with a sequence of graphic pictures showing a woman whose throat had been cut (Safer, Christianson, Autry, \& Österulund, 1998). In yet another study, spider phobics were brought into the laboratory and shown live spiders (Wessel \& Merckelbach, 1997), and so on.

This catalog of gruesome stimuli raises questions about whether we have studied memory for emotional events or, instead, whether we have simply studied memory for shocking or horrifying events. We emphasize that this robs the prior studies of none of their value; these studies inform us in important ways about how certain emotional events are remembered. What is at issue, though, is whether these studies inform us about emotional memory in general (as these earlier researchers often seems to imply) or whether they inform us, instead, about emotional memory for just a certain type of event.

Why might these studies not generalize? One suggestion begins with the idea that the emotional arousal we 
experience in our day-to-day lives is often induced not by a particular visual stimulus, but instead by involvement and empathy with an unfolding event. We become emotional, in other words, when we encounter issues and information that are pertinent to our lives, goals, and values (or perhaps, the lives and values of people we care about). We refer to this kind of emotion as thematically induced, in contrast to the visually induced reactions involved in virtually all of the previous studies. (For more on this distinction, see Laney, Heuer, \& Reisberg, 2003.)

Why should thematically arousing events be remembered differently than visually arousing events? One possibility is that thematic arousal builds gradually, whereas visually induced arousal arrives abruptly (when the unexpected gruesome stimulus jumps into view); it seems plausible that this will influence how someone distributes his or her attention during the event, with consequences for how he or she remembers the event. A different possibility is that thematic arousal, once it arrives, is likely to be sustained, whereas visual arousal is tied to a brief moment in time (when the gruesome stimulus is visible); again, this could influence the allocation of attention and, hence, memory.

We would regard a third possibility, though, as the most pressing: Recall that the Easterbrook (1959) claim attributes participants' narrowed memory to arousal, with the implication that this narrowing would be observed with any stimulus that induces arousal. An alternative, however, is that this narrowing is observed only when an event provides a highly salient stimulus to focus on, a strong attention magnet that seizes participants' visual attention and, therefore, dominates the participants' memories. If this is correct, memory narrowing would be expected if an emotional event contained such an attention magnet but not otherwise, and thus would be expected with visually induced arousal but not with thematically induced arousal.

This suggestion parallels a point raised in a related literature-namely, studies of the so-called weaponfocus effect. This term refers to the fact that victims to crimes involving weapons often seem to focus their attention on the weapon - to the exclusion of all other inputs. As a result, these victims often fail to remember forensically crucial aspects of the crime, including what the perpetrator looked like! (For a review, see Steblay, 1992.) What produces this effect? Some explanations appeal to the Easterbrook (1959) hypothesis, with the claim that the sight of the weapon produces fear and the arousal associated with the fear causes a narrowing of attention. A different possibility, though, is that the weaponfocus effect has nothing to do with arousal. Instead, witnesses focus on the weapon because it is an unusual, surprising, and very important stimulus. On this view, weapon focus is not a consequence of arousal but simply reflects a sensible set of information priorities.

Experimental studies actually suggest that both of these mechanisms - one hinging on arousal and one hinging on the weapon's visual prominence - play a role in producing weapon focus. In some studies, weapon focus has been observed in the absence of emotion, indicating the importance of the weapon's potency as an attention magnet (Kramer, Buckhout, \& Eugenio, 1990; Maass \& Köhnken, 1989). In addition, several studies speak directly to the claim that weapon focus is observed precisely because the weapon is a surprising and novel stimulus (Mitchell, Livosky, \& Mather, 1998; Pickel, 1998, 1999). Other studies, however, suggest that the strength of the weapon-focus effect increases as arousal increases, indicating that arousal also plays a role (e.g., Peters, 1988).

To the best of our knowledge, this issue has never been raised in the domain of autobiographical memory and, with that, in the absence of a weapon. Hence, we simply do not know whether the pattern of memory narrowing, documented in many prior studies, can be generalized to situations involving thematic arousal. The experiments reported here fill this gap.

\section{EXPERIMENT 1}

For our initial foray into this domain, we created a new set of stimuli, designed to produce an emotional response in undergraduate participants without the use of a shocking or horrible visual stimulus. As in previous research, these stimuli involve a slide sequence plus accompanying narration. Crucially, though, there was nothing in the visual presentation that was in any fashion emotional. Instead, the emotional impact of the stimuli was conveyed through the story itself, contained within the narration.

The stimuli focused on an emotional issue relevant to the lives of our college-age participants - namely, date rape. The slides showed a man and a woman on their first date, in the woman's apartment. The neutral and the emotional sequences each consisted of 22 slides and were identical except for one slide, late in the series. In the neutral narration, the participants heard that the woman was relaxed and happy about the date and that the man was polite and friendly. The sequence ended with the woman calling a friend to report on the pleasant evening. In the emotional version, the participants heard early on that the woman was growing apprehensive about the date, with her fears fueled by a number of warning signals during the early part of the date. These fears turned out to be well-founded, as the man attacked the woman late in the sequence. Before the attack could progress, however, the woman pushed the man away, and the story ended with her calling a friend to discuss her distress.

After a brief retention interval, the participants' memories for this event were assessed, via a series of recognition items. This allowed us, for the first time, to examine the memory impact of thematically induced emotion.

\section{Method}

\section{Participants}

Thirty-three undergraduate students at a state university participated; as compensation, the participants were entered into a lottery, and most received course credit. The participants were randomly assigned to the emotional ( 6 men and 12 women) or the neutral ( 5 men 
and 10 women) condition, with the provision that each condition contain approximately equal numbers of male and female participants.

\section{Stimuli}

The stimulus set consisted of 22 slides, each accompanied by a tape-recorded narration. The participants viewed the slides, projected at a size of $66 \times 99 \mathrm{~cm}$, from a distance of approximately $3 \mathrm{~m}$.

The slides depicted a man and a woman on their first date. The initial slides showed the woman getting ready for the date. Slides 5-10 showed the arrival of the man and their early conversation. Slides 11-13 showed a sequence in which they ate dinner, cleared the table, and washed the dishes. Slides 14-17 showed their afterdinner conversation, their good-byes, and the man walking out the door. Slides 18-22 showed the woman locking the door, changing clothes, and calling a friend.

The emotional and the neutral slide sequences were identical, except for one slide. For the participants in the neutral condition, this slide depicted the man and the woman lightly hugging each other, whereas the participants in the emotional condition saw the man in a more aggressive position. His right hand was on her left shoulder, his left hand was holding her wrist, and he appeared to be pushing her backward onto the couch.

Each slide was accompanied by a tape-recorded narration describing the unfolding events of the date. The narration was recorded in a male voice on one track of a stereo tape recorder; the other track contained timing pulses, to ensure synchronization of the narration and the slides. The narration for each slide was followed by $2 \mathrm{sec}$ of silence, and the slide remained in view during this time.

The narration for the emotional group included a number of elements intended to signal the likelihood of an assault. The man was depicted as overbearing, and the story focused on the woman's increasing nervousness as the date progressed. Several predictors of sexual assault were also written into the script, including the consumption of alcohol and a depiction of the man as having an adversarial and disrespectful attitude toward women. For example, the narrative made it clear that the man ignored the woman's opinions, interrupted when she tried to talk, and invaded her personal space.

The neutral narration, in contrast, depicted the man as pleasant and the interactions between the two as comfortable. None of the assault predictors were present in this version. The man and the woman drank fruit juice instead of wine, and the man gave the woman's ideas full consideration and treated her as an equal participant in their conversations. Beyond these crucial differences, though, the narratives for the two conditions were matched as closely as possible (e.g., for sentence structure, length, and so on).

Pilot tests confirmed that the emotional stimulus was, in fact, emotional. Pilot participants simply viewed one or the other stimulus and rated it on a number of dimensions, including how it made them feel. The participants who viewed the emotional stimulus gave reliably higher ratings in response to questions about how angry the stimulus made them feel and, also, how restless and how nervous. They gave reliably lower ratings in response to questions about how amused. Clearly, the stimuli did have the intended emotional effect.

\section{Materials and Apparatus}

During the main experiment, the participants' heart rates were monitored, to determine whether the stimulus did in fact arouse them. For this purpose, a CIC heart rate speedometer was clipped to the participant's earlobe during the stimulus presentation. The speedometer gave a digital output that was recorded by the experimenter at 10 -sec intervals.

Memory test. Following a 20 -min retention interval, the participants completed a memory test for the slide presentation. The test consisted of 91 four-alternative forced choice (4AFC) items (adapted from Rios, 1994). The items were presented in a fixed order, since some of the later questions referred to earlier ones.

The questions were categorized into three types, with the categorization done by two independent judges. (Disagreements were settled by discussion.) Gist questions referred to elements in the story line, rather than to the specific contents of the slides (e.g., "The tape tells you that the woman is . . looking forward to the evening" [incorrect answers: "feeling tired," "nervous," "disinterested in the evening"]). Questions about central details probed memory for plot-irrelevant details that happened to be associated with plot-central objects or players within the slides (e.g., "From the bag she is removing ... a loaf of bread," [incorrect answers: "a bottle of dish soap," "a head of lettuce," "a bunch of carrots"]). Questions about peripheral details, in contrast, focused on details of objects truly in the background of the slide sequence (e.g., "In the background you can see ... a table." [incorrect answers: "a television," "a rocking chair," "a bedroom"]).

In addition, the central and the peripheral detail questions were screened to ensure that central details were visually close to the likely focus of attention within each slide and that peripheral details were far from the likely focus. To this end, a panel of eight judges viewed the slides (four viewed the emotional slides, four the neutral slides) and identified up to three attention centers for each slide: The judges were asked to identify objects or positions within the slides that seemed particularly important and particularly likely to catch someone's attention in viewing the slides. Positions or objects identified by at least three of the four judges were then designated as likely foci of attention. Any point within $4^{\circ}$ of visual angle was considered to be close to participants' probable focus of attention; points further than $4^{\circ}$ were considered far from this focus. (The $4^{\circ}$ radius was largely arbitrary but was guided by considerations of the falloff in parafoveal visual acuity. We have also analyzed the data employing other radii; these analyses indicated that nothing hinges on the $4^{\circ}$ cutoff.)

Note, therefore, that central and peripheral details were distinguished in two ways: Central details concerned attributes of plotrelevant objects or events and were also located close to the probable focus of attention in each slide. Peripheral details concerned plot-irrelevant objects and were far from the probable focus of attention. These two dimensions turn out to be correlated: Not surprisingly, the likely foci identified by the judges corresponded well with the objects we had identified, a priori, as plot relevant. Similarly, the judges tended to ignore those objects we had identified as plot irrelevant. Nonetheless, this double classification of central and peripheral items allowed us to be confident that we had, in fact, identified details at the event's periphery and those truly at the event's center.

The test questions for the neutral and the emotional conditions were matched as fully as possible. In fact, with just one exception, the questions themselves were identical for the two conditions. However, the correct answer was, in several instances, different for the neutral and the emotional conditions.

Secondary measures. Prior to stimulus presentation, each participant was asked to fill out a brief questionnaire on dating practices. This was intended to prime the participants' memories for past dating experiences, so that the participant would be more likely to view the slide presentation from a personal perspective. The expectation was that this personalization would, in turn, increase levels of arousal in the emotional condition. The questions asked in this questionnaire, however, were innocuous, to ensure that the participants would not be alarmed or aroused prior to the stimulus presentation. For example, the questionnaire asked the participants to estimate the number of dates they had per month, to rate the percentage of dates that ended in physical intimacy, and to rate their general feelings toward blind dates.

After viewing the slides, the participants completed Mehrabian's (1977) Stimulus Screening Measure, the trait section of Spielberger's State-Trait Anxiety Inventory (STAI; Spielberger, Gorsuch, \& Lushene, 1970), and a series of rebus problems. These questionnaires were intended primarily to prevent postevent rehearsal of the slide materials, but in addition, these measures provided information that we thought might be predictive of memory performance. 
The results indicate, however, that neither the STAI nor the Screening test was predictive of any of our main measures, and so the questionnaire data will not be considered further.

After completing the memory test, the participants were asked to fill out a posttest questionnaire. This consisted of questions about the participant's immediate reactions to and evaluations of the stimulus presentation. The participants were also asked to rate how strongly they felt each of a list of emotions while watching the stimulus presentation.

\section{Procedure}

All the participants were told that the purpose of the experiment was to measure physiological reactions to various stimuli and that their heart rate would be monitored by a heart rate speedometer. They were also informed that there would be a series of questions to answer before and after the slide presentation.

After completing the consent form, the heart rate monitor was clipped to an earlobe, and the participant was given the dating practices questionnaire to fill out. This provided a time period during which each participant's heart rate could settle to a baseline level. Baseline heart rates were recorded after the participant finished the questionnaire and before the slide presentation began (i.e., approximately 4 min after the clip had been attached).

Following this procedure, the participants viewed either the neutral or the emotional slide presentation. The stimulus presentation lasted approximately $8 \mathrm{~min}$. Each slide was projected for the duration of the tape-recorded sentences accompanying it, plus an additional $2 \mathrm{sec}$. The slides therefore varied in their presentation time, with the shortest being approximately $7 \mathrm{sec}$ and the longest being approximately $26 \mathrm{sec}$. This variation provided a more natural, less monotonous unfolding of the story. Crucially, the duration of the individual slides was matched between the neutral and the emotional versions of the story. (A subsidiary analysis explored the relation between each slide's duration and the participants' memory for the materials in that slide; no correlation was observed.) The interslide interval was approximately $300 \mathrm{msec}$.

After the slide presentation, the participants were asked to remove the ear clip and were given three forms to complete: the Stimulus Screening Measure, the anxiety inventory, and a set of rebus problems. The participants were given as much time as they needed for the first two of these and then were allowed to work on the rebus problems so that the entire retention interval lasted $20 \mathrm{~min}$. At the end of this interval, the participants were told that the experiment was actually concerned with memory for the slide presentation, and they were given the memory test to fill out. (This was the first indication to the participants that this was a memory experiment.) The test took approximately $25 \mathrm{~min}$ to complete. After finishing the memory test, each participant completed the posttest questionnaire and was fully debriefed. The entire procedure lasted approximately $1 \mathrm{hr}$.

\section{RESULTS}

\section{Heart Rate}

Baseline heart rates ranged widely, from 42 to 110 beats per minute (bpm). Within this broad range, baselines for emotional and neutral participants were quite similar, within six bpm (75.7 vs. 81.9 mean bpm). There were no reliable differences in baseline rates between conditions or between sexes.

Given this wide variation in baseline and the absence of a condition difference in baseline, the heart rate data were analyzed as percentage of change from baseline (i.e., each participant's baseline heart rate was subtracted from the rest of his or her recorded heart rates, and all were divided by that participant's baseline). The data pattern is shown in Figure 1, and the pattern suggests that the emotional group was, in fact, aroused by the date rape story. However, although this pattern is qualitatively just what we would expect, any reliable differences were apparently masked by the enormous variability in the participants' heart rates, and the analysis (condition $\times$ sex $\times$ phase) shows no reliable effects.

\section{Subjective Emotionality}

Although the heart rate data are equivocal with regard to the emotional impact of the stimuli, the self-report data are clear. The clearest difference in emotional response between the two conditions was in anger, with the participants in the emotional group giving a mean rating of 2.8 (on a 5-point scale) in response to a question about

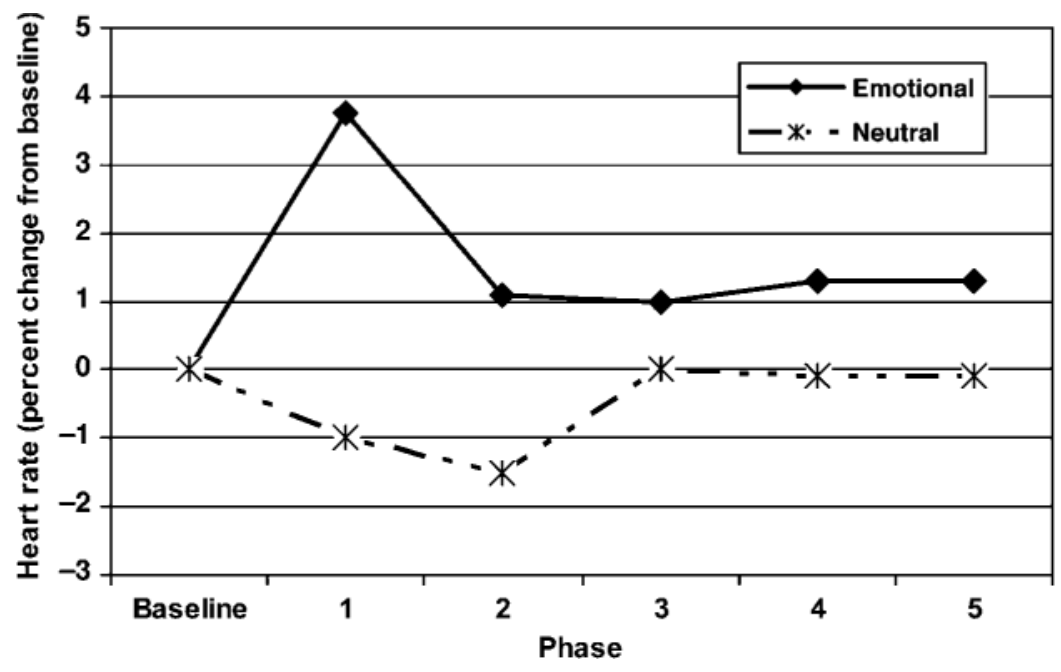

Figure 1. Mean heart rate for emotional and neutral participants across phases of the slideshow, computed as percentage of change from baseline. 
how angry the slides made them feel, a rating reliably higher than that given by the participants in the neutral group [mean of $1.5 ; F(1,31)=6.67, M S_{\mathrm{e}}=1.76, p<$ $.05]$. The participants who viewed the emotional stimulus also rated themselves as more upset than did the participants who viewed the neutral stimulus [means of 2.8 and 1.6; $\left.F(1,31)=5.68, M S_{\mathrm{e}}=1.76, p<.05\right]$.

\section{Memory for the Slide Sequence}

For purposes of analysis, the slide sequence (and the corresponding memory items) was divided into five phases, with each of the phases capturing a step of the narration (preparing for dinner, the conversation before dinner, eating and clearing dinner, after-dinner conversation, and after the date). Nothing marked the phases from the perspective of the participants, but the main story manipulation was in Phases 2-4.

The participants' performance on the memory test was examined in a $2 \times 2 \times 5 \times 3$ analysis of variance (ANOVA), with factors of condition (emotional vs. neutral), sex of participant, phase of presentation, and type of material (gist vs. central details vs. peripheral details). The analysis showed a reliable effect of condition $\left[F(1,29)=6.26, M S_{\mathrm{e}}=4,584.15, p<.02\right]$ and of type of material $\left[F(2,58)=137.59, M S_{\mathrm{e}}=50,093.96, p<\right.$ $.0001]$ and also a reliable interaction between these two factors $\left[F(2,58)=3.80, M S_{\mathrm{e}}=1,383.12, p<.05\right]$. The data are shown in Figure 2. Unsurprisingly, memory for gist is superior to memory for central details, and both are superior to memory for peripheral details. In addition, the main effect of condition reflects the fact that the emotional group had an across-the-board advantage; the interaction, however, reflects the fact that this advantage was largest in remembering the story's gist.

There was also a reliable effect of phase of presentation $\left[F(4,116)=2.96, M S_{\mathrm{e}}=1,120.89, p<.05\right]$, and this effect interacted with type of material $[F(8,232)=$ $\left.3.36, M S_{\mathrm{e}}=1,150.28, p<.01\right]$. In general, this interaction reflects the fact that memory for detail (central vs. peripheral) was somewhat worse in later phases than in earlier ones; this pattern across phases was not observed for memory for gist.

The interaction between phase and condition was near reliable $\left[F(4,116)=2.29, M S_{\mathrm{e}}=866.83, p<.07\right]$. There was also a reliable three-way interaction among phase, condition, and sex of participant $\left[F(4,116)=2.49, M S_{\mathrm{e}}=\right.$ 944.16, $p<.05]$ and a reliable four-way interaction among phase, condition, sex of participant, and type of material $\left[F(8,232)=2.74, M S_{\mathrm{e}}=937.09, p<.01\right]$. Broadly put, the interaction reflects the fact that the emotional story held the participants' interest across all five phases. The neutral story held the female participants' interest across the phases, but not the males'. This pattern was most clearly evident in the participants' memory for gist and their memory for peripheral details, accounting for the four-way interaction.

\section{DISCUSSION}

Thematically induced arousal does seem to influence memory, but the form of this influence may be different from that observed with visually induced arousal. Both forms of arousal promote memory for an event's gist. However, visually and thematically induced arousal may differ with regard to their effects on detail memory. As we noted at the outset, a number of studies have been performed to examine memory for details within a visually arousing sequence (e.g., a sequence involving a bloody face or a sequence involving views of a surgical procedure). These studies have reliably documented a narrowing in participants' recollection of these events, an effect that emerges as an advantage for participants who witnessed an emotional event in remembering materials

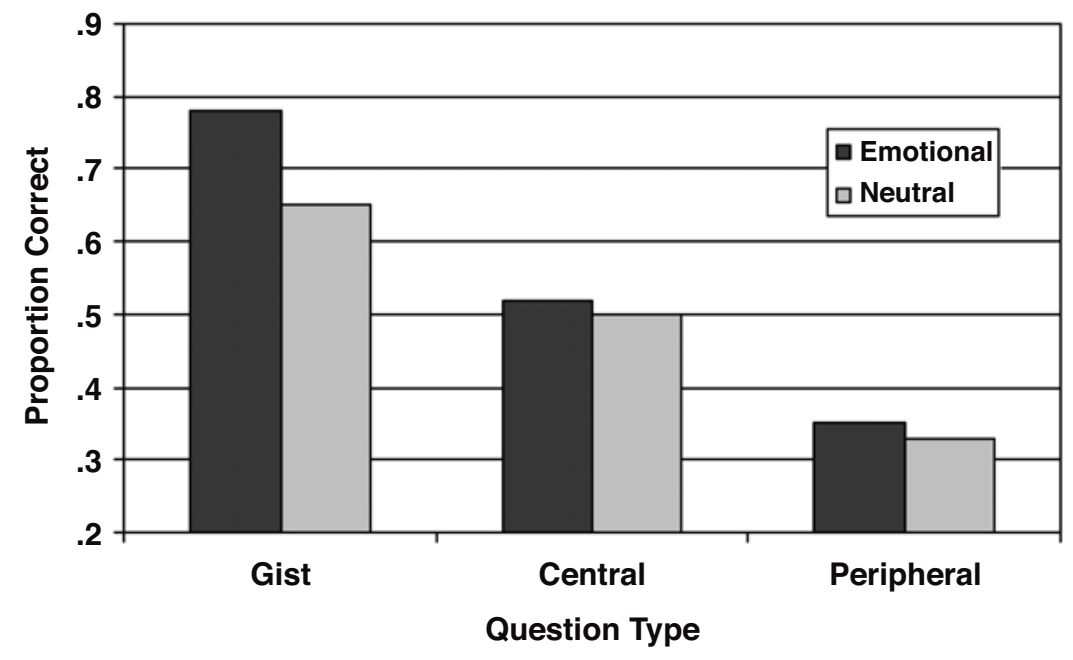

Figure 2. Memory performance of emotional and neutral participants for the three types of to-be-remembered material, averaged across phases. 
central to that event and a disadvantage for these same participants in remembering peripheral aspects of the event. (In both cases, this is in comparison with participants who witnessed a neutral but otherwise comparable event.)

The present results with thematically induced arousal show no indication of this narrowing. That is, we did not observe a disadvantage for the recollection of peripheral aspects of the emotional event. In fact, we observed the opposite - namely, that peripheral details (like all other aspects of the stimulus) were better remembered for the emotional event.

Taken at face value, then, these results indicate that Easterbrook-style narrowing may not be a general feature of emotional remembering. Instead, we would suggest that participants narrow their attention only when an event gives them some striking visual target to focus on-a distinctive or important object, visible within the scene. This target then serves as an attention magnet, and it is this magnet, rather than arousal per se, that causes the observed narrowing of memory.

However, these conclusions must be regarded with caution. The present stimuli obviously involve thematically induced arousal, whereas other studies have employed visually induced arousal. But the present stimuli also differ from those in earlier studies in other ways, and this does allow alternative accounts of the data. In addition, there are, as we noted earlier, other reasons beyond the notion of attention magnets for why a visually arousing event might be remembered differently than a thematically arousing event.

To remove these various possibilities, we would, in the ideal, want to compare stimulus sequences that are identical in all regards except for the fact that one stimulus involves visually induced arousal and the other involves thematically induced arousal. Better still, we would want to compare stimulus sequences that are identical except for the fact that one contains conspicuous attention magnets, whereas the other does not. In our view, though, these comparisons may not be possible, because visual and thematic arousal are, simply, created by different stories and different situations. Therefore, a stimulus involving thematically induced arousal will inevitably differ in several ways from a stimulus involving visually induced arousal, and so perfect matching of stimuli, in order to allow a fully "clean" comparison between these two forms of arousal, may not be attainable.

Perhaps the best way to proceed, therefore, is to work toward an accumulation of data based on a diverse set of stimuli. This accumulation will, in essence, allow us to average across the various stimulus differences and, in this fashion, isolate the variables of interest. The second experiment was undertaken to address this need.

\section{EXPERIMENT 2}

Our second experiment was designed to explore the generality of the first experiment's results. To this end, virtually every aspect of the procedure was altered. As in the first experiment, the neutral and the emotional groups were each shown a series of still photographs, with each accompanied by a narration. This time, however, the photographs were presented on a computer screen, rather than as projected images. Most important, Experiment 2 used an entirely new stimulus set: For both the neutral and the emotional conditions, the stimuli described a day in the life of a college student. In the emotional version of the set, the student had a number of substantial problems and was feeling sufficiently overwhelmed by them to be contemplating suicide; in the neutral version, her life was happier, and there was certainly no possibility of suicide.

As in Experiment 1, this new stimulus set contained no visually exciting elements. Indeed, in the second experiment, there were no visual differences whatsoever between the emotional and the neutral versions of the stimuli: Exactly the same 33 photographs were used for both versions; only the narrations were different.

In addition, a number of the other details of the experiment were also changed. The relatively short retention interval (20 min) used in Experiment 1 was replaced with a 2-day interval. In place of the independent judges who, in Experiment 1, had helped us categorize materials as central or peripheral, a preliminary experiment was conducted using an eye tracker to determine how the participants scanned each photograph. Still other changes in Experiment 2 included a uniform 10-sec presentation time for each photo, rather than a variable 7- to 26-sec presentation time, and the use of an appreciably more fine-grained memory assessment.

\section{METHOD}

\section{Participants}

Thirty-eight students from a small college, all between the ages of 18 and 25 years, were recruited for this study. Each was run individually and spent $15-20 \mathrm{~min}$ in the lab on each of two separate occasions, 2 days apart. Thirty-four participants, 21 females and 13 males, completed all experimental procedures, and only data from these participants are reported. As compensation, the participants were entered into a lottery. Group assignment was quasirandom, with exceptions made to distribute the sexes evenly and to exclude participants with high Beck Hopelessness Scale scores from the emotional group (as described below). In the end, 17 participants (10 females and 7 males) were assigned to the neutral group, and 17 (11 females and 6 males) were assigned to the emotional group. The participants were initially told that they would participate in a study of perception, so that the memory test on their second visit to the lab was unexpected.

\section{Stimuli}

The stimulus set used in this experiment portrayed a day in the life of a college student. In the neutral version, the college student was generally content, doing well in school and having a birthday. In the emotional version, she was depressed, failing out of school, and consequently contemplating suicide. In both cases, attempts were made to draw the viewer into the stimuli via use of secondperson language.

The stimulus set included 33 images, and these were identical for the neutral and the emotional stories. Moreover, the narrations were designed so that, if attention was drawn to a certain visual element 
in the emotion condition, attention was similarly drawn in the neutral condition. Likewise, every effort was made to match the narratives with regard to language and content (e.g., complexity, familiarity, plausibility, and also phrasing). In addition, each slide was presented for $10 \mathrm{sec}$, which allowed for a complete narration for each slide, to develop the story fully and maximize the chances of involving the participants in the story.

The first scene of the slideshow (Slides 1-9) showed Megan, a college student, sitting in her school's dining hall at lunch. The participants were asked to imagine that Megan was a friend of theirs. Megan talks to "you" about either how her life is busy but happy (neutral version) or how miserable she is and how everything is going all wrong (emotional version). In the second scene (Slides 10-13), "you" and Megan walk back to the dorm together. She talks about either how it's her birthday tomorrow (neutral) or about the details of her long-time boyfriend recently ending their relationship (emotional). The third scene (Slides 14-23) takes place the next morning. "You" stop by Megan's room either to wish her a happy birthday (neutral) or to check on her (emotional) and find her either with a hangover (neutral) or feeling suicidal (emotional). In the final scene (Slides 24-33), additional people from the dorm are brought in either to help celebrate Megan's birthday (neutral) or convince her that suicide is not the answer (emotional). At the end of the slideshow, Megan is left alone, either talking to her boyfriend about their evening plans (neutral) or being reassured by her mother (emotional). ${ }^{2}$

\section{Materials and Apparatus}

Memory test. Each of the two versions of a memory test (one per condition) contained 67 4AFC questions. Questions probed for the story's gist and for detailed information relating either to the slides themselves or to the narrations that accompanied the slides.

Before generating any of the questions for the memory test, we laid out a categorization scheme for to-be-remembered materials and then designed questions to fit within this scheme. The categorization scheme defined five types of questions: Both narration gist and visual gist questions described in broad terms the unfolding of the story, with narration gist questions asking about the overall story as described within the narrative and visual gist questions asking, in a general fashion, about the content and layout of the photographs. Narration detail questions probed memory for details of the (tape-recorded) narration, including questions about what was said and by whom.

A fourth and fifth category concerned plot-irrelevant details within the visual stimuli. Visual close detail questions concerned details that happened to be near the likely focus of attention within each slide. Visual far detail questions probed for details about objects or characters that were neither important for the plot nor spatially central within the photographs. This distinction between close and far details was initially done a priori but then was supported by a pilot study, in which 8 participants ( 4 each for the neutral and the arousal stories) viewed the stimuli while their eye positions were recorded (using an ISCAN Pupil/Corneal Reflection Tracking System, ISCAN RK-620PC). Visual inspection of the eye movement patterns made it immediately apparent where the foci of attention were in each picture, and in virtually all cases, the identity of these foci was unsurprising: For example, the participants in both conditions focused primarily on the human faces in each photograph (and on Megan's face in particular). In addition, a small number of other targets reliably caught the pilot participants' view-for example, a telephone that was prominently mentioned in the narratives and also a visually conspicuous fire hydrant. In all cases, though, these eye movement records guided our definition of close and far details, with close details uniformly near the pilot participants' line of view and far details uniformly distant from it.

With these five categories of to-be-remembered items defined in advance, we created questions for the memory test that would represent each of the categories. It was not possible to create equal numbers of questions in each category. (It is the nature of gist that only a few gist questions were possible for each slide, whereas many detail questions were possible.) However, we endeavored to create enough questions in each category that we would have adequate measurements for each type of to-be-remembered material. Table 1 shows the number of questions in each of the five categories.

The majority of the questions (48 of 67 cases) in all categories were identical for the emotional and the neutral group participants, as were the proffered answers. For other questions (10 of 67), the question itself was identical across groups, but the correct answer was not (e.g., "Everyone ends up in Megan's room because . ..," [correct answer: she is threatening suicide for the emotional group and it's her birthday for the neutral group]). In a final group of questions ( 9 of 67), both the question and the answer were different for the groups-generally because there was a need to assess information that was relevant only to one group. Although they were different, even these questions were matched as closely as possible.

\section{Secondary Measures}

Beck Hopelessness Scale. Before viewing the stimuli, the participants were asked to fill out a true/false questionnaire containing 38 questions. These included the 20 questions of the Beck Hopelessness Scale (Beck, Weissman, Lester, \& Trexler, 1974), presented in the original order. Additional items included items from the Life Orientation Test (Scheier, Carver, \& Bridges, 1994) and Mehrabian's (1977) stimulus screening scale.

These questionnaires were intended, first, to screen our participants, in order to avoid showing the suicide story to participants who might themselves be potentially suicidal. For this reason, the 5 participants who scored six or higher on the Beck scale were assigned to our neutral condition, and at the completion of the procedure, the experimenter discussed with them their Beck score and its implications. (We note that this reassignment of participants should work against our experimental manipulations: removing the most

Table 1

Five Categories of Memory Questions Used in Experiment 2

\begin{tabular}{|c|c|c|c|}
\hline Question Type & Number of Questions & Spatial Location & Example Question ... and Answer \\
\hline Narration gist & $9(\mathrm{E}) ; 8(\mathrm{~N})$ & $\mathrm{n} / \mathrm{a}$ & Megan is feeling ... upset. (E only) \\
\hline Narration detail & 17 & $\mathrm{n} / \mathrm{a}$ & Megan's boyfriend is named ... Steve. \\
\hline Visual gist & $9(\mathrm{E}) ; 10(\mathrm{~N})$ & whole scene & $\begin{array}{l}\text { When you find Mark, he is ... in a } \\
\text { kitchen. }\end{array}$ \\
\hline Visual close detail & 13 & central & The RA has . . . a goatee. \\
\hline Visual far detail & 19 & peripheral & $\begin{array}{l}\text { On Megan's feet there are ... cartoon } \\
\text { character slippers. }\end{array}$ \\
\hline
\end{tabular}

Note-E, emotional group; N, neutral group. 
responsive participants from the emotional group, and placing these - perhaps hyperreactive - participants in the neutral group. In any case, this screening of participants was required by our IRB.)

In addition, these scales were intended to prime the participants so that they would easily relate Megan's experiences to their own lives. In this way, we hoped to maximize the effect of the emotion manipulation.

Because the Beck Scale was used to exclude potentially suicidal individuals from the emotional group, there was, inevitably, a reliable difference between the emotional $(M=2.53, S D=1.3)$ and the neutral $(M=4.59, S D=2.9)$ participants on this dimension $[t(22.9)=3.24, p<.01] .^{3}$ These differences, however, did not influence the memory data (e.g., there are no correlations between Beck scores and any of our memory measures, and those individuals who scored over the cutoff score of 6 on the Beck Hopelessness Scale did not perform differently on the memory test than those neutral group participants not meeting this criterion $[t(15)=0.63]$. Thus, this measure will not be discussed further.

Manipulation check. After viewing the stimuli, the participants were asked (via questionnaire) to report their emotional and evaluative reactions to the stimuli. Questions asked for overall judgments of how emotionally engaging and realistic the stimuli were and, also, to what extent the participants had felt various emotions in response to the stimuli.

\section{Procedure}

The participants were brought into the lab twice, 2 days apart. The 1st day's procedure for each participant involved preliminary measurements and the presentation of one of the stimulus sets; the 2nd day was for the surprise memory test.

On the 1 st day, a Cateye PL-6000 heart rate monitor was attached to the participants' earlobes, and baseline heart rates were recorded. The participants gave informed consent and then were given a demographic questionnaire and the Beck Hopelessness Scale to complete. The latter was scored by one of the investigators immediately upon completion. The participants were then divided into groups (emotional and neutral) quasirandomly, with exceptions made to equalize gender and exclude those with high Beck Hopelessness scores from the emotional group. All of this provided approxi- mately $10 \mathrm{~min}$ of sitting time before the stimuli began, allowing heart rates to reach baseline levels.

The participants then viewed either the neutral or the emotional version of the slideshow. Both versions of the stimulus set were presented on a 15 -in $(38-\mathrm{cm})$ iMac DV screen, using the software program iMovie 1.0. The participants were seated at a table approximately $60 \mathrm{~cm}$ from the iMac screen. Each slide was presented for $10 \mathrm{sec}$ with $2 \mathrm{sec}$ interstimulus intervals, for a total presentation time of $6 \mathrm{~min}, 38 \mathrm{sec}$. The narrations were also delivered by the iMac (through its internal speakers), and were recorded (in a male voice) as the soundtrack on the iMovie. The narrations for each slide began as each picture became visible and lasted for the majority of the slide presentation. As the participants watched, their heart rates were recorded at the start of each new slide, or every $12 \mathrm{sec}$.

After the presentation of the slideshow, the participants completed the manipulation check questionnaire. When they had finished it, the participants were excused but were asked to return in 2 days. When they returned, the participants were handed the surprise memory test and were escorted to a quiet private location to complete it. After the participants had completed the memory test, they were debriefed.

\section{RESULTS}

\section{Heart Rates}

As in Experiment 1, baseline heart rates ranged widely-here, from 55 to $118 \mathrm{bpm}$. Within this range, baselines for the emotional and the neutral participants were not significantly different (76.3 vs. 78.8 mean $\mathrm{bpm})$. As in the first experiment, therefore, the heart rate data were analyzed as a percentage of change from baseline. Figure 3 shows the pattern of the data and indicates that our attempt at arousal was successful, with an apparently large difference between the groups. However, and still echoing the pattern in Experiment 1, this seemingly large difference was masked in the analysis by the

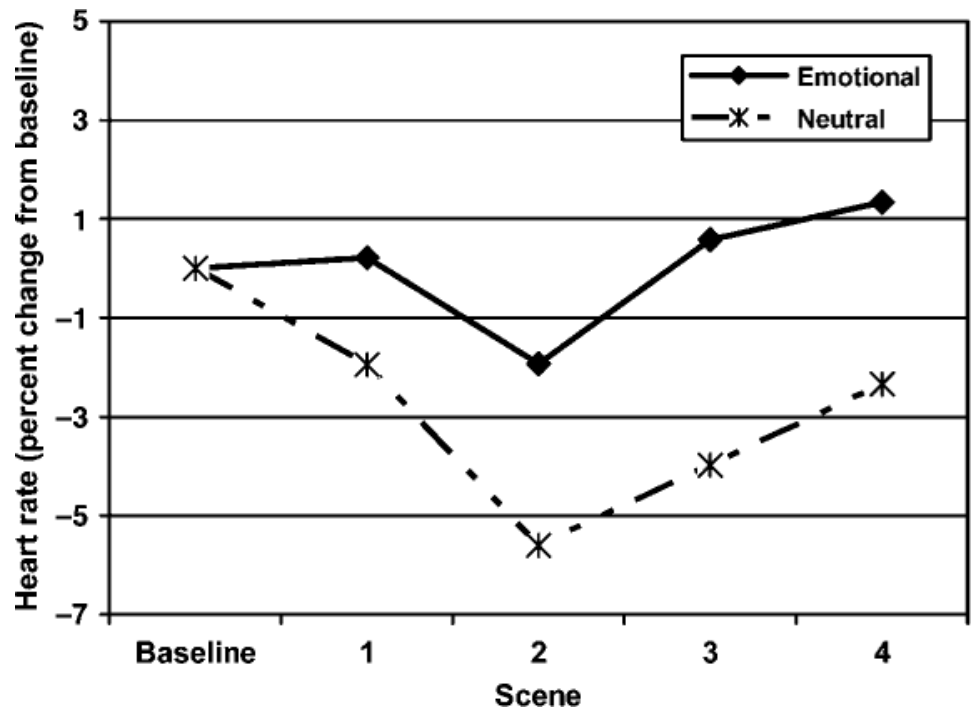

Figure 3. Mean heart rate for emotional and neutral participants across scenes of the slideshow, computed as percentage of change from baseline. 


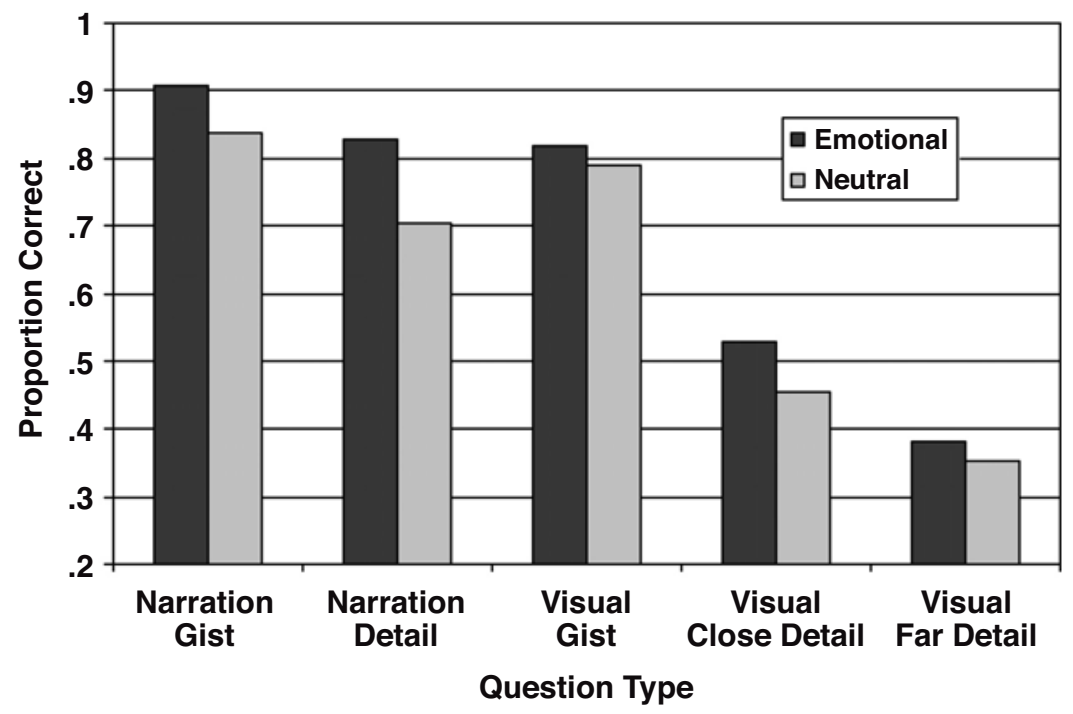

Figure 4. Proportion of memory questions answered correctly as a function of question type and experimental group (emotional vs. neutral).

considerable variability, and so the data do not reveal reliable heart rate differences between the groups.

\section{Subjective Emotionality}

The participants' self-reports, however, paint a much clearer picture of the impact produced by these stimuli. Overall, the participants in the emotion condition found the stimuli to be more emotionally arousing than did those in the neutral condition. In response to the question "Were the slides emotionally engaging for you?" the mean rating for the emotion group (4.38 on a 7 point scale, $S D=1.02$ ) was significantly higher than that for the neutral group $[2.84, S D=1.19 ; t(32)=4.10, p<$ $.001]$. Likewise, the emotional group gave significantly higher ratings $(M=4.27$, on the same 7 point scale, $S D=1.4)$ than did the neutral group $(M=2.53, S D=$ $1.3)$ to the question "How well do you think the presentation told an emotional story?" $[t(32)=3.75, p<.001]$. There was no difference in how the two groups rated the realism of their respective stories $[t(32)=0.57$, n.s.].

In response to the story, the participants in the emotional group rated themselves as feeling more anxious, sad, angry, and nervous than did those in the neutral group. The neutral group rated themselves as more bored and happy than did the emotional group. There were no significant differences between the groups with respect to their reports of feeling relaxed or calm (see Table 2).

\section{Memory for the Slide Sequence}

The participants' performance on the memory test was examined in a $2 \times 2 \times 5$ ANOVA, with factors of condition (emotional vs. neutral), sex of participant, and question type (narration gist vs. narration detail vs. visual gist vs. visual central detail vs. visual peripheral detail). The data are shown in Figure 4, and the analysis revealed a reliable effect of condition $[F(1,30)=7.36, p<$
$.02]$ and of question type $[F(4,120)=108.20, p<.001]$. As in Experiment 1, the emotional story was better remembered than the neutral story, and memory for either visual or narration gist was superior to memory for details. In addition, memory for visual close details was better than that for visual far details.

Importantly, though, there was not a reliable interaction between condition and question type $[F(4,120)=$ 1.48 , n.s.], with the strong suggestion that emotionality benefited all memory categories equally. No other results in this analysis were reliable.

\section{Effects of Expectations}

As an incidental check on whether our surprise memory test was, in fact, unexpected, all the participants were

Table 2

Mean Ratings (on 7-Point Scales) for Eight Separate Emotions by Emotional and Neutral Group Participants $(n=34)$ in Experiment 2

\begin{tabular}{lllll}
\hline Emotion & Group & $M$ & $S D$ & $t(32)$ \\
\hline Anxious & emotional & 4.24 & 1.1 & $2.69^{*}$ \\
& neutral & 2.88 & 1.8 & \\
Sad & emotional & 4.06 & 1.7 & $3.10^{* *}$ \\
& neutral & 2.24 & 1.7 & \\
Bored & emotional & 2.53 & 1.4 & $-3.03^{* *}$ \\
& neutral & 4.00 & 1.5 & \\
Angry & emotional & 2.00 & 0.9 & $4.41^{* *}$ \\
& neutral & 1.00 & 0.0 & \\
Nervous & emotional & 3.29 & 1.4 & $3.16^{* *}$ \\
& neutral & 2.00 & 1.0 & \\
Happy & emotional & 2.00 & 1.2 & $-2.13^{*}$ \\
& neutral & 3.00 & 1.5 & \\
Relaxed & emotional & 3.85 & 1.4 & -1.16 \\
& neutral & 4.47 & 1.7 & \\
Calm & emotional & 3.23 & 1.8 & -1.29 \\
& neutral & 4.00 & 1.6 & \\
\hline
\end{tabular}

$* p<.05 . \quad * * p<.01$. 
asked before they started the memory questions whether they had expected to have their memories tested. Just 3 participants claimed they had: 2 in the neutral group and 1 in the arousal group. An additional participant in the arousal group failed to answer the question. If these 4 participants are removed from the calculations, the results do not change. That is, the main $2 \times 2 \times 5$ ANOVA still yields main effects of condition $[F(1,26)=6.23$, $p<.02]$ and question type $[F(4,10)=96.42, p<.001]$. In addition, there was still no interaction [and so, emotion seemed still to have benefited all question types equally; $F(4,104)=1.33$, n.s.].

\section{GENERAL DISCUSSION}

The results of these experiments provide an initial glimpse of the memory effects of thematically induced, as distinguished from visually induced, emotional arousal. The stimuli and procedures used in Experiment 2 were different in nearly every detail from those in Experiment 1 . The stimuli used different subject matter, different actors, different exposure times, and different numbers of slides - to name just a few of the contrasts. Despite these differences, the two experiments yielded essentially the same results. In both experiments, the emotional group outperformed the neutral group in remembering the broad gist and central details of their respective stimulus sets, showing, for the first time, that the memory advantages associated with emotion can be demonstrated with thematic, as well as visual, emotion. Second, both experiments indicated that these memory advantages extended to all information within the emotional event, so that, in both data sets, emotion promoted memory for both central and peripheral materials. Thus, both experiments failed to show any hint of the memory narrowing associated with emotion in virtually all the previous studies in this domain.

Why do the present data run contrary to the oftenobserved pattern of memory narrowing? We earlier noted that there are several possibilities: Memory may be influenced differently by gradually building arousal (as in the present case) than it is by abruptly arriving arousal (as in previous research). Memory may be influenced differently by arousal that is stretched out in time, in contrast to the effects of a temporally discrete arousal stimulus. Or memory may simply be influenced differently by empathy (as in the present case) than it is by shock or horror (as in previous research).

Our suggestion, though, is different: We suggest that prior studies in this literature do an excellent job of characterizing what happens to memory when someone's attention is caught, during an emotional episode, by a salient visual target. More specifically, the gruesome sights used in prior studies to arouse participants may have served as attention magnets, seizing the viewers' visual attention during the event and dominating their memories afterward. On this logic, and contrary to the
Easterbrook (1959) claim, memory narrowing is not the inevitable by-product of arousal. Instead, memory narrowing is observed when the arousal stimulus itself shapes and narrows attention. Thus, memory narrowing should be associated primarily with visually induced, not thematically induced, arousal.

Having voiced this view, however, let us again offer a note of caution: Precisely because these results contradict a long-standing pattern in the existing literature, our data are clearly in need of corroboration with further findings and with additional stimuli. We have published other data (Laney et al., 2003) indicating that thematic arousal is far more common in day-to-day life than visual arousal, and this in turn suggests that prior studies of emotional memory have studied the atypical case. The present experiments give us a first indication of how the more common form of emotional experience is remembered, but further data are certainly needed in this largely unexplored terrain.

\section{REFERENCES}

Adolphs, R., Tranel, D., \& Denburg, N. (2000). Impaired emotional declarative memory following unilateral amygdala damage. Learning \& Memory, 7, 180-186.

Beck, A. T., Weissman, A., Lester, D., \& Trexler, L. (1974). The measurement of pessimism: The Hopelessness Scale. Journal of Consulting \& Clinical Psychology, 42, 861-865.

Burke, A., Heuer, F., \& ReIsberg, D. (1992). Remembering emotional events. Memory \& Cognition, 20, 277-290.

Cahill, L., \& McGaugh, J. L. (1995). A novel demonstration of enhanced memory associated with emotional arousal. Consciousness \& Cognition, 4, 410-421.

Christianson, S.-Å., \& Loftus, E. F. (1987). Memory for traumatic events. Applied Cognitive Psychology, 1, 225-239.

Christianson, S.-Ã., \& Loftus, E. F. (1990). Some characteristics of people's traumatic memories. Bulletin of the Psychonomic Society, 28, 195-198.

Christianson, S.-Å., \& Loftus, E. F. (1991). Remembering emotional events: The fate of detailed information. Cognition \& Emotion, 5, 81108.

EasterbrooK, J. A. (1959). The effect of emotion on cue utilization and the organization of behavior. Psychological Review, 66, 183-201.

Kramer, T. H., Buckhout, R., \& Eugenio, P. (1990). Weapon focus, arousal, and eyewitness memory: Attention must be paid. Law \& Human Behavior, 14, 167-184.

Laney, C., Heuer, F., \& Reisberg, D. (2003). Thematically-induced arousal in naturally-occurring emotional memories. Applied Cognitive Psychology, 17, 995-1004.

Loftus, E. F., \& Burns, T. E. (1982). Mental shock can produce retrograde amnesia. Memory \& Cognition, 10, 318-323.

MaAss, A., \& KöHNKEN, G. (1989). Eyewitness identification: Simulating the "weapon effect." Law \& Human Behavior, 13, 397-408.

Mehrabian, A. (1977). A questionnaire measure of individual differences in stimulus screening and associated differences in arousability. Environmental Psychology \& Nonverbal Behavior, 1, 89-103.

Mitchell, K. J., Livosky, M., \& Mather, M. (1998). The weapon focus effect revisited: The role of novelty. Legal \& Criminological Psychology, 3, 287-303.

Peters, D. P. (1988). Eyewitness memory and arousal in a natural setting. In M. Gruneberg \& P. E. Morris (Eds.), Practical aspects of memory: Current research and issues (Vol. 1, pp. 89-94). New York: Wiley.

PiCKEL, K. L. (1998). Unusualness and threat as possible causes of "weapon focus." Memory, 6, 277-295. 
PICKEL, K. L. (1999). The influence of context on the "weapon focus" effect. Law \& Human Behavior, 23, 299-311.

Reisberg, D., \& Heuer, F. (2004). Memory for emotional events. In D. Reisberg \& P. Hertel (Eds.), Memory and emotion (pp. 3-41). New York: Oxford University Press.

RIos, C. (1994). The effects of thematically-induced emotion on memory: A spatial narrowing of attention. Unpublished bachelor's thesis, Reed College.

Safer, M. A., Christianson, S.-Å., Autry, M. W., \& Österulund, K. (1998). Tunnel memory for traumatic events. Applied Cognitive Psychology, 12, 99-117.

ScheIER, M. F., CARver, C. S., \& Bridges, M. W. (1994). Distinguishing optimism from neuroticism (and trait anxiety, self-mastery, and self-esteem): A reevaluation of the Life Orientation Test. Journal of Personality \& Social Psychology, 67, 1063-1078.

Spielberger, C. D., Gorsuch, R. L., \& Lushene, R. E. (1970). Psychological and biological approaches to emotion. Hillsdale, NJ: Erlbaum.

Steblay, N. M. (1992). A meta-analytic review of the weapon focus effect. Law \& Human Behavior, 16, 413-424.

Wessel, I., \& MercKelBaCh, H. (1997). The impact of anxiety on memory for details in spider phobics. Applied Cognitive Psychology, 11, 223-231.

\section{NOTES}

1. Easterbrook's (1959) original claim was cast in terms of arousal. Most researchers in this area, however, have focused on the memory effects of emotion. These are, of course, related ideas, on the simple argument that emotionality is usually accompanied by psychological and physiological arousal. However, there is little evidence to indicate whether the memory-narrowing pattern is, in fact, best attributed to emotion or to arousal; this is a topic on which further work is plainly needed.

2. Reviewers of an earlier version of this article expressed concern that the neutral stimulus was not, in truth, neutral, but affectively positive. The stimulus did, after all, mention Megan's birthday and the fact that her relationship with her boyfriend was going well. However, we took care in the stimulus to mention these elements only in a muted way, and, in any case, the neutral status of this stimulus is confirmed by the data: As we will describe in the Results section, the participants who viewed the neutral stimulus, according to their self-report, found it relatively unemotional and, indeed, somewhat boring.

3. Because of unequal variance in the two conditions (as determined by a Levine's test), the degrees of freedom for this calculation have been adjusted from 32 .

(Manuscript received November 12, 2003;

revision accepted for publication February 13, 2004.) 